\title{
ERROR BOUNDS FOR GALERKIN'S METHOD FOR MONOTONE OPERATOR EQUATIONS
}

\author{
MARTIN H. SCHULTZ ${ }^{1}$
}

Abstract. An abstract theorem, generalizing a result of Nitsche, is proved. This gives sharp error bounds for the Galerkin method for approximating the solutions of a large class of nonlinear operator equations in Hilbert spaces.

Let $H$ be a real Hilbert space and $T$ be a strongly monotone operator on $H$ in the sense of Browder, i.e.,

$$
\left|(T u-T v, u-v)_{H}\right| \geqq \gamma\|u-v\|_{H}^{2}
$$

for all $u, v \in H$ and some constant $\gamma>0$. We are interested in numerically approximating the solution of the problem of finding $u \in H$ such that

$$
T u=f, \text { where } f \text { is given in } H,
$$

by the Galerkin method. Given a finite-dimensional subspace, $S$, of $H$, the Galerkin method is to find $u_{S} \in S$ such that

$$
\left(T u_{S}, y\right)_{H}=(f, y)_{H}, \text { for all } y \in S .
$$

From [1] and [2], we recall the following result.

THEOREM 1. If $T$ is uniformly Lipschitz continuous for hounded arguments, i.e., given $B>0$, there exists a positice constant, $C(B)$, depending only on $B$, such that $\|T w-T v\|_{H} \leqq C(B)\|w-v\|_{H}$ for all $w, v \in H$ with $\|w \cdot\|_{I I} \leqq B$ and $\|v\|_{I I} \leqq B$, then problems (2) and (3) have unique solutions and

$$
\left\|u-u_{S}\right\|_{H} \leqq \gamma^{-1} C\left(\|f-T 0\|_{H}\right) \inf _{y \in S} \| u-y:{ }^{\prime}
$$

In many applications, $H$ is a closed subspace of $W^{m .2}(\Omega)$ for some $m \geqq 1$ and (4) yields an error bound in the $W^{m, 2}$-norm when we are really interested in an error bound in the $L^{2}$-norm. While the bound in (4) does induce an error bound in the $L^{2}$-norm, one might expect that such a bound is not sharp and indeed that is the case. In this note, we present an

Received by the editors July 20, 1971 and, in revised form, December 10, 1971.

AMS 1969 subject classifications. Primary 6566, 6580, 6562.

Key nords and phrases. Galerkin method, error bounds, monotont operator.

1 The preparation of this manuscript was supported in part by the Office of Naval Research (N000 14-67-A-0097-0016). 
abstract theorem, generalizing a technique of Nitsche, cf. [3] and [4] for linear selfadjoint problems, which when applied to the problems in question yields sharp $L^{2}$-error bounds directly. See [5] for another such generalization.

Let $V$ and $W^{r}$ be two real Hilbert spaces such that $V \subset H \subset W$ and there exists a positive constant, $K$, such that

$$
\|h\|_{H} \leqq K\|h\|_{I I}, \quad \text { for all } h \in H .
$$

As a concrete example, one may take $H \equiv W_{0}^{m, 2}(\Omega) . V \equiv W^{2 m, 2}(\Omega) \cap$ $W_{0}^{m, 2}(\Omega)$, and $W^{\prime} \equiv L^{2}(\Omega)$.

Instead of (2), we consider the problem of finding $u \in H$ such that

$$
(T u, \phi)_{I I}=(g, \phi)_{W^{*}}, \quad \text { for all } \phi \in H,
$$

where $g$ is given in $W$. Because of (5), problem (6) is a special case of problem (3).

Our new result is

THEOREM 2. Let $C$ be a collection of finite-dimensional subspaces, $S$, of $H$ such that if $u_{\mathrm{s}}$ denotes the Galerkin approximation io $u$ in $S$, then there exist $0<\lambda \leqq \Lambda$ independent of $S$ in $C$ and a bilinear form $b_{S}$ on $H$ such that

(i) $\left(T u-T_{u_{S}}, \phi\right)_{I I}=b_{S}\left(u-u_{S}, \phi\right)$ for all $\phi \in H$ and all $S \in C$,

(ii) $b_{S}(\phi, \phi) \geqq \lambda\|\phi\|_{H}^{2}$ for all $\phi \in H$,

(iii) $\left|b_{S}(w, l)\right| \leqq \Lambda\|w\|_{H}\|v\|_{H}$ for all $w, v \in H$,

(iv) if $b_{S}\left(1,, \phi_{S}\right)=\left(g, w^{\prime}\right)_{W}$ for all $w \in H$, then there exists a positive constant, $\rho$, independent of $S$ in $C$, such that $\|\phi\|_{V} \leqq \rho g \|_{W}$, and

(v) there exists a positice function, $E$, on $S$ such that

$$
\inf _{y \in S} g-\vdots_{\|} \leqq E(S)\|g\|_{V^{\prime}}, \text { for all } S \in C \text { and all } g \in V .
$$

Then

$$
\left\|u-u_{S}\right\|_{H} \leqq \gamma{ }^{1} \rho C^{2}\left(\|f-T 0\|_{H}\right) E(S) \inf _{y \in S}\|u-y\|_{H} .
$$

Proor. For each $S \in C$, let $e_{S} \equiv u-u_{S}$ and consider the problem of finding $\phi_{S} \ldots H$ such that

$$
h_{i j}\left(n \cdot \phi_{S}\right)=\left(e_{S} /\left\|e_{S}\right\|_{W}, w\right)_{W}, \quad \text { for all } w \in H
$$

By our hypotheses on $b_{S}$, this problem has a unique solution, $\phi_{S}$, and $\left\|\phi_{S}\right\|_{r} \leqq \rho$.

Setting $u=c_{3}$. we have $\left\|e_{S}\right\|_{u}=b_{S}\left(e_{S}, \phi_{S}\right)=\left(T u-T u_{S}, \phi_{S}\right)$. Moreover, by the definition of the Galerkin method, we have

$$
e_{S}=\left(T u-T u_{S}, \phi_{S}-y\right)_{H}, \quad \text { for all } y \in S \text {. }
$$


Thus,

$$
\left\|e_{S}\right\|_{W} \leqq C\left(\|f-T 0\|_{H}\right)\left\|u-u_{S}\right\|_{H}\left\|\phi_{S}-v_{\|}\right\|_{H}, \text { for all } y \in S .
$$

Using Theorem 1 to bound $\left\|u-u_{S}\right\|_{H}$ and (7), we ubtain the required result. Q.E.D.

The reader is referred to [6] for further details and applications of this result to boundary value problems for linear and semilinear elliptic partial differential equations and eigenvalue problems.

\section{REFERENCES}

1. F. E. Browder, Approximation-solvability of nonlinear functional equations in normed linear spaces, Arch. Rational Mech. Anal. 26 (1967), 33-42. MR 36 \#3185.

2. P. G. Ciarlet, M. H. Schultz and R. S. Varga, Numerical methods of high-order accuracy for nonlinear boundary value problems. V. Monotone operator theory, Numer. Math. 13 (1969), 51-77. MR 40 \#3730.

3. J. Nitsche, Ein Kriterium für die Quasi-Optimalität des Ritzschen Verfahrens, Numer. Math. 11 (1968), 346-348. MR 38 \#1823.

4. - Verfahren von Ritz und Spline Interpolation hei Sturm-Liouville-Randwert problemen, Numer. Math. 13 (1969), 260-265.

5. - Konvergenz des Ritz-Galerkinschen Verfahrens bei nichtlinearen Operatorgleichungen, International Series of Numerical Mathematics, vol. 15, 1970, pp. 75-81.

6. M. H. Schultz, $L^{2}$-error bounds for the Rayleigh-Ritz-Galerkin method, SIAM J. Numer. Anal. 8 (1971), 737-748.

Department of Computer Science, Yale University, New Haven, Connecticut 06520 